\title{
Enfoque oral en las programaciones curriculares del curso de inglés en las instituciones educativas de jornada escolar completa
}

\author{
Oral focus on the curricular programs of the english course in the \\ educational institutions of Puno full school day
}

\author{
Francisco Marino Tipula Mamani', Vilma Valeriana Tapia Ccallo ${ }^{2}$ \\ ${ }^{1}$ Universidad Nacional del Altiplano-Perú, e-mail: francisco.tipula@gmail.com \\ ${ }^{2}$ Universidad Nacional de Juliaca-Perú, e-mail: vtapia@unaj.edu.pe
}

Recibido e1: 18/10/2018
Aceptado e1: 27/04/2019

\section{RESUMEN}

Enfoque oral en las programaciones curriculares del curso de inglés en las instituciones educativas de jornada escolar completa, considera el proceso de interacción dinámica entre uno o más interlocutores para comunicar sus ideas y emociones en el aula a partir de la lectoescritura para la enseñanza del inglés; este proceso es inadecuado, ya que el propósito del estudio es evaluar la aplicación del enfoque oral en las programaciones curriculares del curso de inglés. La metodología es de carácter descriptivo evaluativo en la que se analizó 17 sesiones de aprendizaje programadas para el período académico 2017 del primer grado de educación secundaria, para describir los tipos de actividades que incluyan contenidos de entonación, acentuación y ritmo. El resultado de la evaluación de 231 actividades programadas en un $23 \%$ se aproxima para desarrollar la oralidad. En conclusión, la aplicación del enfoque oral en las programaciones curriculares del curso de inglés en los colegios JEC., es escasa la programación de actividades de entonación, acentuación y no se advierte actividades de ritmo para promover la oralidad del idioma inglés.

Palabras clave: enfoque oral en inglés, entonaciónacentuación-ritmo.

\section{ABSTRACT}

Oral approach in the curricular programming of the English course in the educational institutions of full school day, considers the process of dynamic interaction between one or more interlocutors to communicate their ideas and emotions in the classroom from the literacy for the teaching of English; which is an improper procedure. The purpose of the study is to evaluate the application of the oral approach in the curricular programming of the English course. The methodology is of an evaluative descriptive nature in which 17 scheduled learning sessions were analyzed for the 2017 academic period of the first grade of secondary education, to describe the types of activities that include intonation, accentuation and rhythm contents. The result of the evaluation of 231 programmed activities in a $23 \%$ is close to develop orality. In conclusion, the application of the oral approach in the curricular programming of the English course in JEC schools, there is little programming of intonation activities, accentuation and rhythm activities are not noticed to promote the orality of the English language.

Keywords: oral focus in English, intonationaccentuation-rhythm. 


\section{INTRODUCCIÓN}

El enfoque oral en las programaciones curriculares del curso de inglés en las instituciones educativas de jornada escolar completa, considera el proceso de interacción dinámica entre uno o más interlocutores para comunicar sus ideas y emociones en el aula a partir de la lectoescritura para la enseñanza del inglés. Las razones fundamentales para promover la oralidad es hacer uso de la lengua para comunicarse; sin embargo, la oralidad en las programaciones curriculares está como ausentes. Casi todas las actividades programadas para una sesión de clases en inglés inician con la lecto-escritura.

La enseñanza de la pronunciación es una asignatura pendiente Villaescusa (2009). Puesto que, la identidad geográfica, social y cultural de una persona se percibe a través de sus características fonológicas individuales. Al respecto Blanco (2012), indica que en los programas académicos se aprecia la falta de atención prestada al componente fónico en la enseñanza de lenguas extranjeras. Y el componente fónico para Quilis (1981), es ideal para transmitir las más diversas informaciones, que en el proceso de comunicación van mezcladas y el oyente decodifica automáticamente, y sabe si su interlocutor pregunta, afirma o niega.

La enseñanza de la entonación es una actividad pendiente en aulas de lengua extranjera. Molina (2014), la entonación es fundamental en el discurso oral y su incorporación en las actividades orales; para acelerar procesos de adquisición de la pronunciación en lengua extranjera; por otro lado, Becerra (2012), manifiesta que los rasgos de la pronunciación se asocian con el tono de voz, intensidad y duración de enunciados. El éxito de la comunicación inicia con la entonación, al respecto García (2016), afirma que las principales manifestaciones del lenguaje son la oralidad y la entonación en la comunicación; porque son dos aspectos fonéticos adquiridos en la lengua materna. Para enseñar la entonación desde una perspectiva comunicativa conversacional Hidalgo (2015), plantea estrategias metodológicas como: escucha y repite; observa y visualiza la curva melódica; audición simultánea del sonido; corrige y perfecciona con el objetivo de experimentar una práctica activa del estudiante.

El valor de la oralidad del inglés, de acuerdo con Cantero (2015), los interlocutores negocian el valor de su timbre, las variantes de cada consonante, el ritmo del habla, la entonación prelingüística (es decir, su acento), y el valor de las inflexiones tonales (lingüístico y paralingüístico). En esa negociación tácita radica el valor de la oralidad. A esto suma Poch (2004), afirmando que el aprendizaje de la pronunciación (sonido, entonación, etc.) debe ocupar un espacio en el proceso de adquisición de lengua extranjera y Usó (2008), advierte que la preferencia por el acento extranjero responde a un posicionamiento real y es fruto de una actitud lingüística.

Asimismo, Ahumada (2010), determina que usar una lengua es saber hablarla; aunque la atención a ella ha sido relegada de la enseñanza de segunda lengua (L2) durante largo tiempo, lo cual se observa en la práctica docente actual. Del mismo modo Llisteri (2003), manifiesta: la pronunciación es una de las destrezas que todo alumno necesita dominar cuando aprende una lengua extranjera. Entonces, la no priorización de la oralidad Blanco (2012), genera problemas de integración en el grupo, en la comunidad y la participación social.

Por tanto, el MINEDU (2018), establece que la comunicación oral en inglés en el aula es una interacción dinámica entre uno o más interlocutores para comunicar sus ideas-emociones. La cual es una buena intensión ministerial; que no se traduce en la práctica docente del curso de inglés; porque la oralidad sigue ausente en las programaciones y actividades del docente del curso de inglés. El objetivo de investigación planteado es evaluar la aplicación del enfoque oral en las programaciones curriculares del curso de inglés de las Instituciones Educativas de Jornada Escolar Completa. 


\section{MARCO TEÓRICO}

\section{El enfoque oral}

La importancia del enfoque oral radica en la interacción dinámica de los interlocutores, Giralt (2014), el enfoque oral es la opción metodológica para la enseñanza-aprendizaje de la pronunciación de segunda lengua extranjera (enseñanza de la lengua extranjera E/LE.); está basada en la acción, donde los aprendices desarrollan una lengua con fluidez y corrección. Asimismo para Hernandez (2014), la comprensión auditiva es un proceso interactivo que consiste en percibir y construir mensajes mediante un sinnúmero de mecanismos cognitivos y afectivos porque es esencial entender lo que otros dicen para poder contestar o responder a otra persona. Y Otero (2012), enfatiza que en el éxito comunicativo es clave la entonación y los rasgos suprasegmentales de la lengua. De modo que, la enseñanza de la pronunciación en los programas curriculares del curso de inglés constituye una tarea urgente en todos los niveles. Es así que Giralt (2014), fundamenta que enseñar a pronunciar es parte del acto de habla como tal, que solo podrá adquirirse una competencia fónica cuando se use la lengua oral en un proceso de comunicación significativa.

\section{Situación actual de la pronunciación en la clase de ELE}

En la actualidad la pronunciación en aula se da en un ámbito interdisciplinario en que los avances tecnológicos Llisterri (2007), deben influir con una mejor comprensión del proceso de adquisición del sistema fonético de una L2. Asimismo, Paredes (2013), la pronunciación implica dos procesos: la percepción y la producción; la percepción es una competencia con un conjunto de conocimientos y habilidades para discriminar los sonidos de la lengua meta; y la producción es una habilidad para poder realizar esos sonidos. Aunque Bartolí (2013), advierte que, existe la tendencia a trabajar poco la pronunciación porque no se sabe muy bien cómo integrarla en la clase comunicativa. Además se presenta desvinculada de la lengua oral, sujeta a la lengua escrita en mayor o menor grado según los manuales. Y los contenidos de pronunciación se introducen en el orden siguiente: sonidos aislados, en contexto, ritmo, acento y entonación.

En relación a la prosodia del habla Hernández (2015), establece: 1). El acento es la sensación perceptiva encargada de realizar el contraste entre las sílabas fuertes y débiles mediante la modulación del tono, la duración y la intensidad. 2). La entonación es el número de vibraciones por segundo de las cuerdas vocales y se asocia con el tono más grave o más agudo; los tonos gramaticales son: enunciativa, interrogativa y exclamativa. Finalmente 3) El ritmo, es la temporalización acentual, para ejercitar se puede emplear canciones infantiles, refranes y frases.

\section{Diseño curricular nacional del curso de inglés}

El Currículo Nacional de la Educación Básica del MINEDU (2017), respecto al perfil de egreso del curso de inglés expone que la comunicación oral en inglés como lengua extranjera es una interacción dinámica entre los interlocutores para comunicar (ideas, emociones) y la comprensión-producción supone un proceso activo de construcción de diversos tipos de textos orales.

Metodología y elaboración de materiales para la pronunciación

Las metodologías para enseñar una lengua extranjera en la escuela es propuesta por Padilla (2007), el método verbo tonal (MVT) esta metodología sigue tres pasos: 1) técnicas relacionadas con la dimensión lúdico-afectiva, 2) técnicas relacionadas con el entrenamiento receptivo y 3) técnicas relacionadas con el entrenamiento productivo.

1) Técnica lúdico-afectiva, es aprender una lengua jugando, así como los niños adquieren su lengua materna, lo cual le ayuda a perder inhibiciones y a lanzarse a la pronunciación. Los ejercicios en aula incluyen cinco factores: motivación, lengua de partida, edad, carácter o personalidad y objetivos. 
2) Técnica de entrenamiento receptivo, es reconocer antes que producir, porque cuando se adquiere la lengua materna; nuestro cerebro se niega a oír los sonidos de otras lenguas. La mejor manera de hacerlo es seguir un camino similar al de los niños que aprenden la L1.

3) Técnicas relacionadas con el entrenamiento productivo, consiste en utilizar ejercicios de diálogos y teatrillos con personajes que interpretarán cada uno de los alumnos. Por ejemplo, diálogos multifuncionales que puedan cambiar su significado en función de valores expresivos o pragmáticos.

Igualmente, Hudson (2013), concibe estrategias para aprender una L2 como: imitación, demostración, asociación, explicación, métodos articulatorios, pares mínimos, audición e imitación, sistema verbotonal. Además, MINEDU (2017), implementa en la Jornada Escolar Completa JEC, el modelo mixto Blended, esto significa, el docente y la tecnología trabajen de forma integrada asumiendo roles específicos. Los roles son las sesiones virtuales donde se desarrolla una interacción estudiantecomputador/software, el estudiante interioriza el idioma empleando una plataforma virtual. Por ejemplo, las sesiones "Face to Face", el trabajo del estudiante es guiado por el docente. Los recursos de enseñanza-aprendizaje están constituidos por un kit

60 de licencias para estudiantes-docentes, manuales, cuadernos, software, etc. Finalmente, Guillén (2012), plantea la pronunciación a través de: 1) el método tradicional, promueve el buen uso la lengua escrita; 2) el método audiolingüe; presenta cada sonido en relación al vocabulario y 3) el método comunicativo, promueve la comunicación oral.

\section{METODOLOGÍA}

\section{Diseño metodológico}

La investigación corresponde al enfoque mixto, de tipo no experimental y es de carácter descriptivo, de acuerdo con Hernández, Fernández, y Baptista (2016), consiste en describir fenómenos, situaciones, contextos y sucesos; es así como se manifiesta el enfoque oral en las programaciones curriculares. Además corresponde al diseño evaluativo Flores (2011), porque en el estudio se recoge información con el fin de hacer estimaciones acerca del valor del contenido, es decir las actividades de entonación, acentuación y ritmo en las programaciones curriculares.

\section{Técnica e instrumento}

Se utilizó la técnica documental, porque se revisó documentos oficiales de las programaciones curriculares del curso de inglés presentadas en el portal web de MINEDU, para las Instituciones Educativas de Jornada Escolar Completa, en esta plataforma virtual está las programaciones de las sesiones de aprendizaje del curso de inglés para el año académico 2017. El instrumento utilizado fue la ficha de información electrónica en la que se recabó la información de los documentos curriculares para identificar la presencia o ausencia de actividades que promuevan los contenidos de la entonación, acentuación y ritmo del inglés.

\section{Unidades de análisis.}

Debido a la naturaleza del estudio y de acuerdo a los resultados de los antecedentes de investigación, se considera importante la enseñanza de la oralidad de la lengua extranjera. Con este propósito esta investigación consideró las actividades de aprendizaje programadas para el curso de inglés por MINEDU (2017), correspondiente al primer grado de educación secundaria del año académico 2017. La unidad de análisis está conformada por 231 actividades de aprendizaje, que están distribuidas como contenido de inicio, desarrollo y cierre de la sesión; en las cuales se analizó la presencia o ausencia de acentuación, la entonación y el ritmo.

\section{Procesamiento de datos}

Realizado el análisis de los contenidos de las actividades programadas en las sesiones de aprendizaje de inglés determinando la presencia o ausencia de contenidos con actividades de entonación, acentuación y el ritmo; para el procesamiento de la información se utilizó el 
programa excel y se organizó en cuadros para la información cualitativa y tablas para los datos cuantitativos considerando la frecuencia y porcentaje.

\section{Variables de estudio}

El enfoque oral es la opción metodológica para la enseñanza-aprendizaje de la pronunciación de la lengua extranjera está basada en la acción, donde los aprendices desarrollan una lengua con fluidez y corrección.

En las programaciones curriculares del curso de inglés en las Instituciones Educativas de Jornada Escolar Completa, se observa, que la variable es el enfoque oral considerada como una opción metodológica para la enseñanza-aprendizaje de la pronunciación de la lengua extranjera está basada en la acción, donde los aprendices desarrollan una lengua con fluidez y corrección. Las dimensiones e indicadores son:

1. La entonación, contenidos que actúe en la frase para darle tono.

2. El acento, contenidos que actúe en la palabra o en las sílabas.
3. El ritmo, contenidos que manifieste la distribución en el tiempo de algunos elementos suprasegmentales, tales como el acento o la silaba

\section{ANÁLISIS DE RESULTADOS Y DISCUSIÓN}

\section{La entonación en las programaciones curriculares}

La enseñanza de la entonación, afirma Hernández (2015), se realiza en tres fases: 1) la educación del oído o percepción de los cambios entonativos, para este propósito se puede programar actividades como pronunciar las siguientes frases: I'm reading a book; Are you reading a book? y I'm not reading a book. 2) la producción guiada por el docente en la que los alumnos sean capaces de repetir enunciados o textos que el docente lleve al aula, en este caso el profesor conduce la pronunciación. Y 3) la producción comunicativa, en la que el alumno sea capaz por sí mismo reproducir variaciones entonativas. En el siguiente cuadro se describe los contenidos de la entonación en actividades programadas para sesiones de aprendizaje del curso de inglés.

\section{Cuadro 1.}

Descripción de actividades de la entonación en las programaciones.

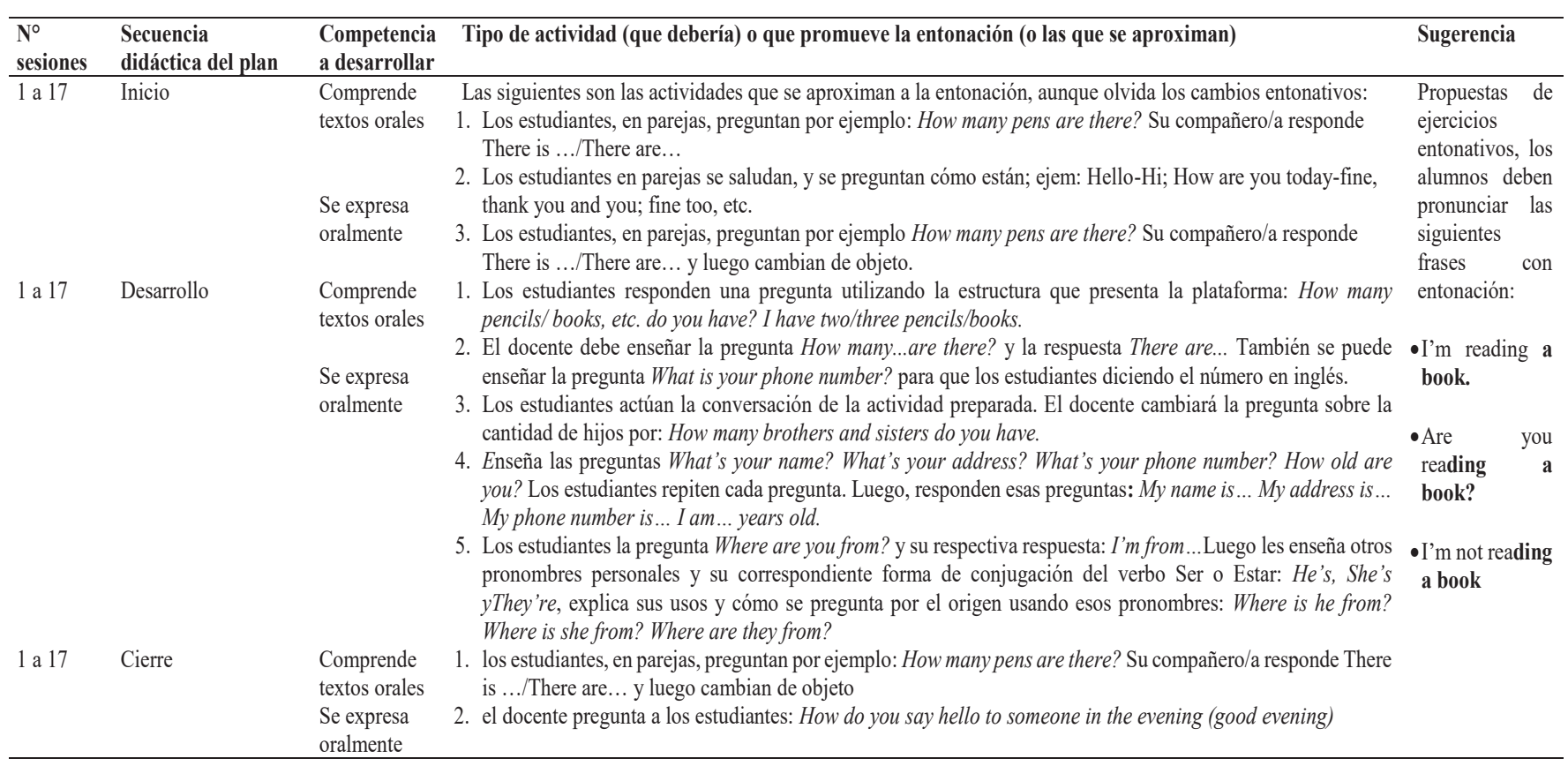


Del cuadro 01 se observa que hay 10 actividades que se aproximan a la oralidad, así como: Los estudiantes, en parejas, preguntan por ejemplo How many pens are there? Su compañero/a responde There is .../There are... Ninguna de las actividades descritas contiene los cambios entonativos. Al respecto Castillo (2016), afirma que la entonación es usar tipos especiales de entonación porque mejora la utilización de un modelo entonativo. Ejemplo: a) Will you, / please? b) It ${ }^{\text {ee }}$ better. En a) la entonación ascendente favorece una pronunciación tensa de $/ \mathrm{i} / . \mathrm{Y}$ en b) el uso de un tipo descendente favorece la pronunciación relajada de / $/$ '".

Tabla 1

Actividades que promueven la entonación en las sesiones programadas.

\begin{tabular}{|c|c|c|c|c|c|c|c|c|}
\hline \multirow{3}{*}{$\begin{array}{l}\mathrm{N}^{\circ} \text { de } \\
\text { sesiones }\end{array}$} & \multicolumn{6}{|c|}{ Actividades en la secuencia didáctica } & \multirow{2}{*}{$\begin{array}{l}\text { Total } \\
\text {-actividades }\end{array}$} & \multirow{2}{*}{$\begin{array}{l}\text { Total } \\
\text { actividades }\end{array}$} \\
\hline & Inicio & & Desarrollo & & Cierre & & & \\
\hline & $\begin{array}{l}\text { Actividades } \\
\text { programadas }\end{array}$ & $\begin{array}{l}\text { actividades } \\
\text { entonación }\end{array}$ & $\begin{array}{l}\text { Actividades. } \\
\text { Programadas }\end{array}$ & $\begin{array}{l}\text { Actividades } \\
\text { entonación }\end{array}$ & $\begin{array}{l}\text { Actividades } \\
\text { de cierre }\end{array}$ & $\begin{array}{l}\text { Actividades } \\
\text { Entonación } \\
\end{array}$ & programas & entonación \\
\hline 1 & 6 & 0 & 19 & 1 & 5 & 0 & 30 & 1 \\
\hline 2 & 3 & 0 & 3 & 0 & 2 & 0 & 8 & 0 \\
\hline 3 & 2 & 0 & 15 & 0 & 3 & 1 & 20 & 1 \\
\hline 4 & 3 & 0 & 3 & 0 & 2 & 0 & 8 & 0 \\
\hline 5 & 5 & 0 & 3 & 0 & 3 & 0 & 11 & 0 \\
\hline 6 & 4 & 2 & 12 & 3 & 4 & 1 & 20 & 6 \\
\hline 7 & 3 & 0 & 3 & 0 & 2 & 0 & 8 & 0 \\
\hline 8 & 3 & 1 & 11 & 3 & 4 & 1 & 18 & 5 \\
\hline 9 & 3 & 1 & 3 & 1 & 2 & 0 & 8 & 2 \\
\hline 10 & 2 & 0 & 3 & 1 & 4 & 1 & 9 & 2 \\
\hline 11 & 6 & 1 & 9 & 1 & 4 & 1 & 19 & 3 \\
\hline 12 & 3 & 1 & 3 & 1 & 2 & 1 & 8 & 3 \\
\hline 13 & 3 & 0 & 13 & 1 & 4 & 0 & 20 & 1 \\
\hline 14 & 3 & 1 & 3 & 1 & 2 & 0 & 8 & 2 \\
\hline 15 & 3 & 0 & 4 & 1 & 3 & 1 & 10 & 2 \\
\hline 16 & 3 & 0 & 11 & 2 & 4 & 0 & 18 & 2 \\
\hline 17 & 3 & 1 & 3 & 0 & 2 & 0 & 8 & 1 \\
\hline Total & 58 & 8 & 121 & 16 & 52 & 7 & 231 & 31 \\
\hline
\end{tabular}

Fuente: elaboración del equipo-programación de sesiones de aprendizaje en inglés IE. JEC. 2017.

62 De la tabla 1 se advierte que en 17 sesiones del curso de inglés existen 231 actividades programadas y solo hay 31 actividades que se aproximan con contenidos de entonación de las cuales 8 están en inicio, 16 en desarrollo y 7 en cierre. Ninguna de estas actividades promueve expresamente la entonación. Al respecto Backhouse (2011), conceptúa la entonación como una función lingüística socialmente representativa y que individualmente refleja diferentes sentidos en una oración; porque, la manera en que se entona una oración puede dar diferentes significados. Ejemplo: vengo (tono descendente) y ¿Vengo? (tono ascendente). Y por ello, es imprescindible poner énfasis en las actividades de entonación a través de ejercicios de comprensión auditiva; asimismo Balterio (2015), destaca que la pronunciación como la percepción de acentos son susceptibles de interaccionar con nuestros estudiantes; así como la producción individual de fonemas, palabras, frases, ritmo, entonación, etc.

\section{La acentuación en las programaciones curriculares.}




\section{Cuadro 2}

Descripción de actividades de la acentuación en las programaciones del curso de inglés.

\begin{tabular}{|c|c|c|c|c|}
\hline $\begin{array}{l}N^{\circ} \\
\text { sesiones }\end{array}$ & $\begin{array}{l}\text { Secuencia } \\
\text { didáctica } \\
\text { del plan }\end{array}$ & $\begin{array}{l}\text { Competencia } \\
\text { a desarrollar }\end{array}$ & $\begin{array}{l}\text { Tipo de actividad (que debería) o que promueven la acentuación (o } \\
\text { las que se aproximan) }\end{array}$ & Sugerencia \\
\hline 1 a 17 & Inicio & $\begin{array}{l}\text { Comprende } \\
\text { textos orales } \\
\mathrm{Se} \text { expresa } \\
\text { oralmente }\end{array}$ & $\begin{array}{l}\text { 1.Después, en parejas, los estudiantes se preguntan How do you spell your } \\
\text { name? y responden, por ejemplo: L-U-I-S, D-A-Y-A-N-A. } \\
\text { 2.Utiliza palabras que les resultan familiares a los estudiantes: apple, bed, } \\
\text { cat, dog, egg, office, rabbit y queen. Reforzar la repetición coral. } \\
\text { 3.El docente escribe en la pizarra una lista de palabras usadas } \\
\text { anteriormente: cat, bed, ring, dog, cup, cake, kite, rope, student, teacher, } \\
\text { book, house, street, train, cow. Los estudiantes las leen en voz alta. Se } \\
\text { verifica la correcta pronunciación de las palabras. }\end{array}$ & $\begin{array}{l}\text { Los estudiantes } \\
\text { pronuncian } \\
\text { conforme al } \\
\text { stress on words. } \\
\text { Una forma de } \\
\text { acentuación } \\
\text { puede ser: }\end{array}$ \\
\hline 1 a 17 & Desarrollo & $\begin{array}{l}\text { Comprende } \\
\text { textos orales } \\
\text { Se expresa } \\
\text { oralmente }\end{array}$ & $\begin{array}{l}\text { 1. Los estudiantes escuchan la pronunciación las palabras anteriores } \\
\text { señalando la primera letra de la palabra en la pizarra mientras se muestra } \\
\text { la imagen. Los estudiantes repiten las palabras. } \\
\text { 2. Por turnos, preguntan y responden: - How do you spell your name? - D- } \\
\text { A-N-I-E-L } \\
\text { 3. Muestra imágenes de cat, bed, ring, dog, cupy las pronuncia enfatizando } \\
\text { las vocales. Los estudiantes pronuncien las palabras. } \\
\text { 4. Los estudiantes practican la pronunciación en parejas y luego, se } \\
\text { comprueba la correcta pronunciación de las respuestas. Luego, les pide a } \\
\text { los estudiantes que traten de agregar una palabra más a cada una de las } \\
\text { categorías de la separata, por ejemplo: shut, choose, thank, ring, clock, } \\
\text { white, knife. }\end{array}$ & $\begin{array}{l}\text { pronunciamos } \\
\text { con mayor } \\
\text { acento la parte } \\
\text { con negrillas: } \\
\text { afternoon, until, } \\
\text { engineer, } \\
\text { result, police, } \\
\text { Halloween, } \\
\text { Image, } \\
\text { Japanene. }\end{array}$ \\
\hline 1 a 17 & Cierre & $\begin{array}{l}\text { Comprende } \\
\text { textos orales } \\
\mathrm{Se} \text { expresa } \\
\text { oralmente }\end{array}$ & $\begin{array}{l}\text { 1. Finalmente, el docente pregunta a los estudiantes y ellos responden: } \\
\text { ¿Which object begins with b? bed; with h? hat; wiht } 1 \text { ? lemon; with t? ten } \\
\text { 2. Para terminar la sesión, el docente muestra imágenes de shoe, cheese, } \\
\text { mouth, ring, white, clock, knife y los estudiantes repiten las palabras }\end{array}$ & \\
\hline
\end{tabular}

Fuente: elaboración del equipo-programación de sesiones de aprendizaje en inglés IE. JEC. 2017.

En el cuadro 2 hay nueve actividades, una de ellas describe: después, en parejas los estudiantes se preguntan How do you spell your name? y responden, por ejemplo: L-U-I-S, D-A-Y-A$\mathrm{N}$-A. Esta actividad promueve la repetición de vocales, consonantes, división silábica, acento de intensidad y a veces el acento ortográfico; basada en la lecto-escritura; pero ninguna promueve la acentuación del idioma inglés. De acuerdo con Hernández (2016), el primer ejercicio que se puede resaltar en clase la actividad de escuchar palabras; por ejemplo, conforme al stress on words, una forma de acentuación puede ser: pronunciamos con mayor acento la parte con negrillas: afternoon, until, engineer, result, police, Halloween, Image, 63 Japanene.

Tabla 2

Actividades que promueven la acentuación en las sesiones de aprendizaje

\begin{tabular}{|c|c|c|c|c|c|c|c|c|}
\hline \multirow{3}{*}{$\begin{array}{l}\mathrm{N}^{\circ} \text { de } \\
\text { sesiones }\end{array}$} & \multicolumn{6}{|c|}{ Actividades en la secuencia didáctica } & \multirow{3}{*}{$\begin{array}{c}\text { Total } \\
\text { actividades } \\
\text { programas }\end{array}$} & \multirow{3}{*}{$\begin{array}{c}\text { Total } \\
\text { actividades } \\
\text { acentuación }\end{array}$} \\
\hline & \multicolumn{2}{|c|}{ Inicio } & \multicolumn{2}{|c|}{ Desarrollo } & \multicolumn{2}{|c|}{ Cierre } & & \\
\hline & $\begin{array}{l}\text { Actividad } \\
\text { programada }\end{array}$ & $\begin{array}{l}\text { actividades } \\
\text { acentuación }\end{array}$ & $\begin{array}{l}\text { Actividades } \\
\text { Programadas }\end{array}$ & $\begin{array}{l}\text { Actividades } \\
\text { acentuación }\end{array}$ & $\begin{array}{l}\text { Actividades } \\
\text { de cierre }\end{array}$ & $\begin{array}{l}\text { Actividades de } \\
\text { acentuación }\end{array}$ & & \\
\hline 1 & 6 & 0 & 19 & 1 & 5 & 1 & 30 & 2 \\
\hline 2 & 3 & 1 & 3 & 0 & 2 & 1 & 8 & 2 \\
\hline 3 & 2 & 0 & 15 & 1 & 3 & 1 & 20 & 2 \\
\hline 4 & 3 & 1 & 3 & 0 & 2 & 0 & 8 & 1 \\
\hline 5 & 5 & 1 & 3 & 0 & 3 & 0 & 11 & 1 \\
\hline 6 & 4 & 1 & 12 & 1 & 4 & 1 & 20 & 3 \\
\hline 7 & 3 & 0 & 3 & 0 & 2 & 0 & 8 & 0 \\
\hline 8 & 3 & 1 & 11 & 0 & 4 & 0 & 18 & 1 \\
\hline 9 & 3 & 1 & 3 & 0 & 2 & 0 & 8 & 1 \\
\hline 10 & 2 & 0 & 3 & 1 & 4 & 0 & 9 & 1 \\
\hline 11 & 6 & 1 & 9 & 1 & 4 & 1 & 19 & 3 \\
\hline 12 & 3 & 1 & 3 & 0 & 2 & 0 & 8 & 1 \\
\hline 13 & 3 & 0 & 13 & 1 & 4 & 0 & 20 & 1 \\
\hline 14 & 3 & 1 & 3 & 0 & 2 & 0 & 8 & 1 \\
\hline 15 & 3 & 0 & 4 & 1 & 3 & 1 & 10 & 2 \\
\hline 16 & 3 & 0 & 11 & 1 & 4 & 0 & 18 & 1 \\
\hline 17 & 3 & 0 & 3 & 0 & 2 & 0 & 8 & 0 \\
\hline Total & 58 & 9 & 121 & 8 & 52 & 6 & 231 & 23 \\
\hline
\end{tabular}

Fuente: elaboración del equipo-programación de sesiones de aprendizaje en inglés IE. JEC. 2017. 
En la tabla 2 se observa que en 17 sesiones existen 231 actividades programadas de las cuales 23 actividades seaproximan a la acentuación. Ejemplo: se escribe shoe, cheese, mouth, ring, white, clock y knife. Luego, los estudiantes pronuncian el sonido del dígrafo consonante; o en parejas se preguntan How many pens are there? Su compañero/a responde There is .../There are... estas actividades promueven la lectura, la escritura, reconocimiento de las vocales y consonantes, etc. Pero ninguna de las actividades mencionadas promueve la acentuación. A ello corrobora Hernández (2015), el acento puede trabajarse desde tres opciones: 1) percepción y sensibilización del acento; 2) imitación y producción del acento, siguiendo normas de acentuación inglesa y 3) producción libre con una correcta acentuación. Aunque Vergara y Perdomo (2016), plantean el andamiaje de escritura creativa que fortaleció la producción oral y escrita en inglés de los estudiantes. Este fortalecimiento se vio reflejado en la fluidez oral, la pronunciación y el uso de la gramática durante las interacciones comunicativas.

Calderón (2015), reconoce que hay la necesidad imperante por aprender una segunda lengua, que en este caso es el inglés. Para tal propósito García (2016), sugiere cuatro pasos:1) método de oposiciones fonológicas, se centra, en la percepción, fase previa a la correcta producción de sonidos. 2) método fonoarticulatorio es la descripción articulatoria de los sonidos de la L2 despierta la atención hacia ellos. 3) método tecnológico se dirige hacia la percepción y la concienciación. Y, 4) el MVT incide directamente en la práctica o producción. Sus procedimientos se relacionan con la disposición de los sonidos de forma que su pronunciación resulte más cómoda.

\section{EI ritmo en las programaciones curriculares.}

Cuadro 3

Descripción de contenidos del ritmo en las programaciones de las sesiones de aprendizaje del curso de inglés.

\begin{tabular}{|c|c|c|c|c|}
\hline $\begin{array}{l}\mathrm{N}^{\circ} \\
\text { sesiones }\end{array}$ & $\begin{array}{l}\text { Secuencia } \\
\text { didáctica } \\
\text { del plan }\end{array}$ & $\begin{array}{l}\text { Competencia a } \\
\text { desarrollar }\end{array}$ & $\begin{array}{l}\text { Tipo de actividad (que debería) } \\
\text { o que promueven el ritmo (o las } \\
\text { que se aproximan) }\end{array}$ & Sugerencia \\
\hline 1 a 17 & Inicio & $\begin{array}{l}\text { Comprende textos orales } \\
\text { Se expresa oralmente }\end{array}$ & $\begin{array}{l}\text { No existe ni por aproximación } \\
\text { actividades que promuevan el }\end{array}$ & $\begin{array}{l}\text { Actividad que } \\
\text { promueva el ritmo }\end{array}$ \\
\hline 1 a 17 & Desarrollo & $\begin{array}{l}\text { Comprende textos orales } \\
\text { Se expresa oralmente }\end{array}$ & ritmo del idioma inglés. & $\begin{array}{l}\text { del idioma inglés, } \\
\text { son las canciones }\end{array}$ \\
\hline 1 a 17 & Cierre & $\begin{array}{l}\text { Comprende textos orales } \\
\text { Se expresa oralmente }\end{array}$ & & $\begin{array}{l}\text { en inglés, } \\
\text { trabalenguas, etc. }\end{array}$ \\
\hline
\end{tabular}

Fuente: elaboración del equipo-programación de sesiones de aprendizaje en inglés IE. JEC. 2017.

En el cuadro 3, se constata que no se programa actividades para promover el ritmo del idioma inglés. Si se deseará trabajar el ritmo de una lengua tenemos el Ejemplo de William Shakespeare: When I do count the clock that tells the time. (Cuando cuento el reloj que da las horas) To be, or not to be, that is the question. (Ser o no ser, esa es la cuestión); Double, double toil and trouble; Fire burn, and caldron bubble. (Doble, doble esfuerzo y problema; el fuego arde y el caldero burbujea). Por lo cual Hernández (2015), considera tres formas para aprender el ritmo: 1) la percepción y la sensibilización del oído a las características del ritmo en español; 2) la producción e imitación por parte del alumno guiado por el docente y 3) la producción libre y practica comunicativa. 
Tabla 3

Actividades que promueven el ritmo del inglés en las sesiones de aprendizaje.

\begin{tabular}{|c|c|c|c|c|c|c|c|c|}
\hline \multirow{3}{*}{$\begin{array}{l}\mathrm{N}^{\circ} \text { de } \\
\text { sesiones }\end{array}$} & \multicolumn{6}{|c|}{ Actividades en la secuencia didáctica } & \multirow{3}{*}{$\begin{array}{l}\text { Total } \\
\text { actividades } \\
\text { programas }\end{array}$} & \multirow{3}{*}{$\begin{array}{l}\text { Total } \\
\text { actividades } \\
\text { de ritmo }\end{array}$} \\
\hline & & \multicolumn{2}{|c|}{ Desarrollo } & \multicolumn{2}{|l|}{ Cierre } & & \\
\hline & $\begin{array}{l}\text { Actividades } \\
\text { programadas }\end{array}$ & $\begin{array}{l}\text { actividades } \\
\text { de ritmo }\end{array}$ & $\begin{array}{l}\text { Actividades } \\
\text { Programadas }\end{array}$ & $\begin{array}{l}\text { Actividades } \\
\text { de ritmo }\end{array}$ & $\begin{array}{l}\text { Actividades } \\
\text { de cierre }\end{array}$ & $\begin{array}{l}\text { Actividades } \\
\text { de ritmo }\end{array}$ & & \\
\hline 1 & 6 & 0 & 19 & 0 & 5 & 0 & 30 & 0 \\
\hline 2 & 3 & 0 & 3 & 0 & 2 & 0 & 8 & 0 \\
\hline 3 & 2 & 0 & 15 & 0 & 3 & 0 & 20 & 0 \\
\hline 4 & 3 & 0 & 3 & 0 & 2 & 0 & 8 & 0 \\
\hline 5 & 5 & 0 & 3 & 0 & 3 & 0 & 11 & 0 \\
\hline 6 & 4 & 0 & 12 & 0 & 4 & 0 & 20 & 0 \\
\hline 7 & 3 & 0 & 3 & 0 & 2 & 0 & 8 & 0 \\
\hline 8 & 3 & 0 & 11 & 0 & 4 & 0 & 18 & 0 \\
\hline 9 & 3 & 0 & 3 & 0 & 2 & 0 & 8 & 0 \\
\hline 10 & 2 & 0 & 3 & 0 & 4 & 0 & 9 & 0 \\
\hline 11 & 6 & 0 & 9 & 0 & 4 & 0 & 19 & 0 \\
\hline 12 & 3 & 0 & 3 & 0 & 2 & 0 & 8 & 0 \\
\hline 13 & 3 & 0 & 13 & 0 & 4 & 0 & 20 & 0 \\
\hline 14 & 3 & 0 & 3 & 0 & 2 & 0 & 8 & 0 \\
\hline 15 & 3 & 0 & 4 & 0 & 3 & 0 & 10 & 0 \\
\hline 16 & 3 & 0 & 11 & 0 & 4 & 0 & 18 & 0 \\
\hline 17 & 3 & 0 & 3 & 0 & 2 & 0 & 8 & 0 \\
\hline Total & 58 & $\mathbf{0}$ & 121 & 0 & 52 & 0 & 231 & 0 \\
\hline
\end{tabular}

Fuente: elaboración del equipo-programación de sesiones de aprendizaje en inglés IE. JEC. 2017.

De la tabla 3 se constata que no existe actividad que promueva el ritmo del idioma inglés, para Hernández (2015), el ritmo es la duración en el tiempo de los diferentes suprasegmentales en función de la sílaba. Para ejercitar el ritmo del inglés se puede usar canciones en inglés según la edad, refranes y otros de manera lúdica los alumnos pueden percibir el ritmo. De la misma forma Silva
(2006), afirma que las canciones de música popular en la enseñanza del inglés facilitan el aprendizaje de la competencia comunicativa, porque motivan y reducen la ansiedad del estudiante. Por otro lado Sanhueza (2005), indica que la internet es un medio eficaz para la enseñanza de la pronunciación inglesa, el cual puede perfectamente combinarse con los métodos clásicos de instrucción.

\section{Cuadro 4}

Enfoque oral en las programaciones curriculares.

\begin{tabular}{|c|c|c|c|}
\hline $\begin{array}{l}\mathrm{N}^{\circ} \\
\text { sesiones }\end{array}$ & $\begin{array}{l}\text { Secuencia } \\
\text { didáctica del } \\
\text { plan }\end{array}$ & $\begin{array}{l}\text { Competencia } \\
\text { a desarrollar }\end{array}$ & $\begin{array}{l}\text { Tipo de actividad (que debería) o que promueve la oralidad (o las que se } \\
\text { aproximan) }\end{array}$ \\
\hline 1 a 17 & Inicio & $\begin{array}{l}\text { Comprende } \\
\text { textos orales } \\
\text { Se expresa } \\
\text { oralmente }\end{array}$ & $\begin{array}{l}\text { 1.Después, en parejas, los estudiantes se preguntan How do you spell your name? y } \\
\text { responden, por ejemplo: L-U-I-S, D-A-Y-A-N-A. } \\
\text { 2.Utiliza palabras que les resultan familiares a los estudiantes: apple, bed, cat, dog, egg, } \\
\text { office, rabbit y queen. Reforzar la repetición coral. } \\
\text { 3.El docente escribe en la pizarra una lista de palabras usadas anteriormente: cat, bed, ring, } \\
\text { dog, cup, cake, kite, rope, student, teacher, book, house, street, train, cow. Los estudiantes } \\
\text { las leen en voz alta. Se verifica la correcta pronunciación de las palabras. }\end{array}$ \\
\hline 1 a 17 & Desarrollo & $\begin{array}{l}\text { Comprende } \\
\text { textos orales } \\
\text { Se expresa } \\
\text { oralmente }\end{array}$ & $\begin{array}{l}\text { 1.Los estudiantes escuchan la pronunciación las palabras anteriores señalando la primera letra } \\
\text { de la palabra en la pizarra mientras se muestra la imagen. Los estudiantes repiten las palabras. } \\
\text { 2.Por turnos, preguntan y responden: - How do you spell your name? }-D-A-N-I-E-L \\
\text { 3. Muestra imágenes de cat, bed, ring, dog, cupy las pronuncia enfatizando las vocales. Los } \\
\text { estudiantes pronuncien las palabras. } \\
\text { 4.Los estudiantes practican la pronunciación en parejas y luego, se comprueba la correcta } \\
\text { pronunciación de las respuestas. Luego, les pide a los estudiantes que traten de agregar una } \\
\text { palabra más a cada una de las categorías de la separata, por ejemplo: shut, choose, thank, } \\
\text { ring, clock, white, knife. }\end{array}$ \\
\hline 1 a 17 & Cierre & $\begin{array}{l}\text { Comprende } \\
\text { textos orales } \\
\text { Se expresa } \\
\text { oralmente }\end{array}$ & $\begin{array}{l}\text { 1.Finalmente, el docente pregunta a los estudiantes y ellos responden: ¿Which object begins } \\
\text { with b? bed; with h? hat; wiht } 1 \text { ? lemon; with t? ten } \\
\text { 2.Para terminar la sesión, el docente muestra imágenes de shoe, cheese, mouth, ring, white, } \\
\text { clock, knife y los estudiantes repiten las palabras }\end{array}$ \\
\hline
\end{tabular}

Fuente: elaboración del equipo - programación de sesiones de aprendizaje en inglés IE. JEC. 2017. 
El cuadro 4 refiere que las actividades propuestas son para completar e identificar vocales, consonantes, división silábica, el acento de intensidad, la entonación de las palabras y a veces el acento ortográfico; basada en la lecto-escritura. Las 231 actividades programadas no enfatizan la oralidad en el aprendizaje del idioma inglés. Sobre ello Aguilar (2012), enfatiza que la competencia comunicativa destaca el carácter funcional de la lengua como instrumento de comunicación, por lo que las funciones, y no los contenidos gramaticales, pasan a ser el elemento que vertebra la enseñanza. Y por función lingüística se entiende aquello que hacemos con la lengua, es decir, dar y pedir información, expresar tristeza o alegría, felicitar, disculparse, dar consejos, etc.

Tabla 4

Evaluación del enfoque oral en las sesiones del curso de inglés

\begin{tabular}{|c|c|c|c|c|c|}
\hline \multirow[b]{2}{*}{$\begin{array}{l}\mathrm{N}^{\circ} \text { de } \\
\text { sesiones }\end{array}$} & \multicolumn{3}{|c|}{ Enfoque oral } & \multirow{2}{*}{$\begin{array}{l}\text { Total de } \\
\text { actividades } \\
\text { que aplica el } \\
\text { enfoque oral }\end{array}$} & \multirow[b]{2}{*}{$\begin{array}{l}\text { Total } \\
\text { actividades } \\
\text { programas }\end{array}$} \\
\hline & $\begin{array}{l}\text { Total } \\
\text { actividades } \\
\text { entonación }\end{array}$ & $\begin{array}{l}\text { Total } \\
\text { actividades } \\
\text { acentuación }\end{array}$ & $\begin{array}{l}\text { Total } \\
\text { actividad } \\
\text { es ritmo }\end{array}$ & & \\
\hline 1 & 2 & 2 & 0 & 4 & 30 \\
\hline 2 & 2 & 2 & 0 & 4 & 8 \\
\hline 3 & 2 & 2 & 0 & 4 & 20 \\
\hline 4 & 1 & 1 & 0 & 2 & 8 \\
\hline 5 & 1 & 1 & 0 & 2 & 11 \\
\hline 6 & 3 & 3 & 0 & 6 & 20 \\
\hline 7 & 0 & 0 & 0 & 0 & 8 \\
\hline 8 & 1 & 1 & 0 & 2 & 18 \\
\hline 9 & 1 & 1 & 0 & 2 & 8 \\
\hline 10 & 1 & 1 & 0 & 2 & 9 \\
\hline 11 & 3 & 3 & 0 & 6 & 19 \\
\hline 12 & 1 & 1 & 0 & 2 & 8 \\
\hline 13 & 1 & 1 & 0 & 2 & 20 \\
\hline 14 & 1 & 1 & 0 & 2 & 8 \\
\hline 15 & 2 & 2 & 0 & 4 & 10 \\
\hline 16 & 1 & 1 & 0 & 2 & 18 \\
\hline 17 & 0 & 0 & 0 & 0 & 8 \\
\hline Total & 31 & 23 & $\mathbf{0}$ & 54 & 231 \\
\hline Porcentaje & 13 & 10 & $\mathbf{0}$ & 23 & 100 \\
\hline
\end{tabular}

En la tabla 4 hay 13\% de contenidos aproximados a las actividades de entonación y $10 \%$ a contenidos de la acentuación. En total el 23\% de actividades son contenidos aproximados a la enseñanza de la entonación y acentuación del idioma inglés. Sepulcre (2012), propone el método verbo tonal (MVT) para aprender el idioma inglés: el MVT., ve problemas de audición; la persona que aprende una lengua extranjera no oye los contrastes fonéticos. La enseñanza de la pronunciación de L2 inicia con la reeducación de la percepción y asimilación de la lengua meta. Corrobora Mendoza (2017), para los estudiantes que aprenden una lengua extranjera, resulta que la expresión oral es la destreza más difícil de aprender. Los estudiantes generalmente presentan diferentes deficiencias como organizar y estructurar el discurso de modo coherente y exponer claramente. Finalmente Cantero (2015), define la importancia de la pronunciación en la comunicación, además de ser un componente lingüístico, es pragmático, sociolingüístico y crucial en las destrezas orales y su influencia en la adquisición de las habilidades lingüísticas, deben estar programadas.

\section{CONCLUSIONES}

Los resultados del estudio permitieron evaluar la aplicación del enfoque oral en las programaciones curriculares del curso de inglés de las Instituciones Educativas de Jornada Escolar Completas logrando describir que las actividades programadas en 
las sesiones de aprendizaje del curso de inglés hay escasa programación de actividades de entonación, acentuación y ninguna en relación a las actividades de aprendizaje del ritmo; así como no existe actividades específicas para promover la comprensión auditiva; que según las teorías y antecedentes de la investigación la ausencia del enfoque oral para aprender el idioma inglés hace difícil el desarrollo eficaz de la expresión oral.

\section{Sugerencias}

De acuerdo a los resultados del estudio, teorías y antecedentes de investigación se sugiere a los responsables en la enseñanza del curso de inglés programar actividades de aprendizaje con contenidos de entonación, acentuación y ritmo para aprender la expresión oral del idioma inglés al $100 \%$; y las actividades de lecto-escritura se pospongan para niveles superiores, es así como se aprende la lengua materna.

\section{CONFLICTOS DE INTERESES}

Los autores declaran no tener ningún conflicto de intereses.

\section{AGRADECIMIENTO}

A la Universidad Nacional del Altiplano, por su apoyo incondicional en la consecución del presente artículo de investigación.

\section{REFERENCIAS BIBLIOGRÁFICAS}

Aguilar, A. (2012). Influencia de la dramtización en la expresión oral de discentes universitarios extranjeros $y$ nativos. Retrieved from http://riubu.ubu.es/bitstream/10259/195/1/ Aguilar_López.pdf

Ahumada, G. P. (2010). La enseñanza de la pronunciación en ELE: Una asignatura pendiente., 138. https:// papyrus.bib.umontreal.ca/xmlui/ bitstream/handle/1866/4508/ Ahumada_Gladys_P_2010 these. pdf? sequence $=2$ \&isAllowed $=y$
Backhouse, Å. (2011). Los principales problemas fonéticos y fonológicos en la enseñanza del español para extranjeros. Retrieved from http://www.diva-portal.org/smash/get/ diva2:519078/FULLTEXT01.pdf

Balterio, I. (2015). La importancia de la pronunciación en la forma-lisis de problemas y necesidades., 275-288.

Bartolí, M. (2013). La pronunciación por tareas en la clase de ELE. Phonica, 910. http://www.tesisenred.net/bitstream/ handle/10803/134881/MBR TESIS. pdf? sequence $=1$

Becerra, M. (2012). Rasgos prosódicos en la producción de dos formas de ironía en Español. Linguistica, 28(1), 191-205. Retrieved from http://www.scielo.edu.uy/ pdf/ling/v28n1/v28n1a10.pdf

Blanco, A. (2012). Adquisición y aprendizaje del componente fónico del Español como lengua extranjera. Rla, 50(2), 13-37.

Calderón, P. (2015). Modelo de negocio online para el aprendizaje del inglés para adultos jóvenes de Hispanoamérica. Retrieved from https://repository.unimilitar. edu.co/bitstream/handle/10654/13953/ finaltesis PaolaCaldeorn.pdf; jsessionid $=65 \mathrm{~A} 2 \mathrm{C} 0834817267$ D69CDBE697F9322BF? sequence $=2$

Cantero, J. (2015). De la fonética del habla 67 espontánea a la fonología de la complejidad from phonetics of spontaneous speech to phonology of complexity, 195, 9-29. https:// doi.org/10.7203/Normas.5.681

Castillo, J. (2016). Mejoramiento de la pronunciación de la lengua inglesa de los estudiantes de primer semestre del programa de lenguas extranjeras de la universidad Santiago de Cali a través de la enseñanza de la fonética. Retrieved from http://bibliotecadigital.univalle.edu.co/ bitstream/10893/9618/1/CB0541274.pdf

Flores, J. (2011). Construyendo la tesis universitaria. (1 $\mathrm{ra}$ ed.). Lima. Impreso en Garden Graf S.R.L.

García, A. (2016). ¿Dónde está la entonación? Propuesta didáctica integradora 
de la entonación en el nivel Al. Retrieved from https://dugi-doc.udg. edu/bitstream/handle/10256/15402/ GarciaCoronadoAndrea Treball. pdf?sequence $=1$

Giralt, M. (2014). El enfoque oral en la adquisición de la pronunciación del E/LE: algunas cuestiones sobre fonética perceptiva. (A. (eds) Congosto, Y., Montero, M. y Salvador, Ed.). Libros.SL.

Guillén,P.(2012). La enseñanza de la pronunciación en el aula de español como segunda lengua: del método tradicional al enfoque comunicativo. Káñina, Revista de Artes $y$ Letras, 36(1), 205-213. Retrieved from http://revistas.ucr.ac.cr/index.php/kanina/ article/view/1233

Hernández, Roberto; Fernández, Carlos \& Baptista, P. (2016). Metodología de la Investigación (6ta ed.).

Hernandez, E. (2014). El B-learning como estrategia metodológica para mejorar el proceso de enseñanza-aprendizaje de los estudiantes de inglés de la modalidad semipresencial del departamento especializado de idiomas de la Universidad Técnica de Ambato. Retrieved from http://eprints.ucm.es/29610/1/T35913.pdf

Hernández, V. (2015). Fonética en ELE: los elementos suprasegmentales y su aplicación en el aula. Retrieved from http://digibuo. uniovi.es/dspace/bitstream/10651/37647/6/ TFM_Hernández Pierna.pdf

Hidalgo, A. (2015). Enseñar la entonación en E/ LE: problemas, desafíos y propuesta de soluciones. Foro de Profesores de E/LE, 11(0). https://doi.org/10.7203/foroele.0.7114

Hudson, R. (2013). Propuestas para la enseñanza de la pronunciación y corrección fonética en español como lengua extranjera. Retrieved from https://cvc.cervantes.es/ensenanza/ biblioteca_ele/publicaciones_centros/PDF/ budapest 2013/04 bueno.pdf

Llisteri, J. (2003). Evaluación de la pronunciación, diseño curricular y Diplomas de Español, 1-13. http://liceu.uab.es/ joaquim/ publicacions/Llisterri 03 Pronunciacion ELE.pdf
Llisterri, J. (2007). La enseñanza de la pronunciación asistida por ordenador, 91-120. Retrieved from http://liceu.uab. cat/ joaquim/publicacions/Llisterri 06 Pronunciacion_Tecnologias.pdf

Mendoza, M. (2017). Estrategias metodológicas para mejorar las habilidades de speaking y listening en idioma ingles en la escuela de educación básica de universidad laica Eloy Alfaro de Manabí Methodological strategies to improve the speaking and listening skills in English, 3, 588-641.

MINEDU. (2017a). Currículo Nacional | Minedu. Retrieved March 13, 2019, from http://www. minedu.gob.pe/curriculo/

MINEDU. (2017b). Manual acompañante ingles. Retrieved March 10, 2019, from https:// es.slideshare.net/lituha/manual-acompaanteingles-49370384

Molina, I. (2014). Entonación, Intención y Relevancia. La importancia de la entonación y su enseñanza en el aula de ELE. Algunas propuestas didácticas. Revista de Didáctica ELE, 19(1885-2211), 1-21.

Otero, H. (2012). Aprender y enseñar entonación en aulas multilingües. XXIII Congreso Internacional de La ASELE, 664-675. Retrieved from http://cvc.cervantes.es/ ensenanza/biblioteca_ele/asele/pdf/23 123 0067.pdf

Padilla, X. (2007). El lugar de la pronunciación en la clase de ELE. XVII Congreso Internacional de La ASELE, 871-887. Retrieved from http:// cvc.cervantes.es/ensenanza/biblioteca_ele/ asele/pdf/17/17 0871.pdf

Paredes,F.(2013).Laenseñanza delapronunciación en Español como Lengua Extranjera: cuestiones de método. Linred-Lingüística en la red monográfico III Jornadas de lengua y 4comunicación. fonética: Enseñanza $e$ investigación, 11., 1-30. Retrieved from http://www.linred.es/monograficos_pdf/LR monografico11-2-articulo3.pdf

Poch, D. (2004). La pronunciación en la enseñanza del Español como lengua extranjera. RedELE: Revista Electrónica de Didáctica de Español Como Lengua Extranjera, 1, 145-152. 
Quilis, A. (1981). Funciones de la entonación Anton io Quil is. Retrieved from https://core. ac.uk/download/pdf/84863655.pdf

Sanhueza, E. (2005). El uso de internet en el aprendizaje de la pronunciación inglesa una experiencia con estudiantes de ingeniería. Retrieved from http://www.tesis.uchile. cl/tesis/uchile/2005/sanhueza_e/sources/ sanhueza_e.pdf

Sepulcre, S. (2012). No es lo que dices, sino cómo lo dices. Foro de Profesores de E/LE, 5, 1-10. Retrieved from https://ojs.uv.es/index. $\mathrm{php} /$ foroele/article/view/6574

Silva, M. (2006). La enseñanza del inglés como lengua extranjera en la titulaci+on defilología inglesa: uso de las canciones de música popular no sexista como recurso didáctico.
Retrieved from http:/www.biblioteca.uma. es/bbldoc/tesisuma/16853805.pdf

Usó, L. (2008). La enseñanza de la pronunciación en LE: algunas consideraciones a tener en cuenta. Phonica, 4(February), 104-130.

Vergara,A., \&Perdomo, M.(2016). Fortalecimiento de la expresión oral y escrita en inglés a través de un andamiaje de escritura creativa colaborativa: un estudio de diseño desde la cognición distribuida. Forma y función, 30(1), 117. https://doi.org/10.15446/fyf. v30n1.62417

Villaescusa, I. (2009). «La enseñanza de la pronunciación en la clase de ELE». Retrieved from http://cvc.cervantes.es/ensenanza/ biblioteca_ele/publicaciones_centros/pdf/ manila 2009/10 investigaciones 03.pdf 